\title{
Noncontact Large-Scale Displacement Tracking: Doppler Radar for Water Level Gauging
}

\author{
Changzhan Gu, Member, IEEE, Wenyao Xu, Member, IEEE, Guochao Wang, Takao Inoue, Member, IEEE, \\ Jennifer A. Rice, Lixin Ran, and Changzhi Li, Senior Member, IEEE
}

\begin{abstract}
Doppler radar has been used for small-scale displacement detection, e.g. respiration and heartbeat. The motion amplitude is less than half a carrier wavelength. In this letter, a novel Doppler radar technique is presented for accurate tracking of large-scale displacement of several carrier wavelengths, e.g., the water level variation. The DC-coupled architecture is used to ensure precise measurement of slow motions even with stationary moment. A novel signal processing approach is proposed to increase the demodulation linearity to deal with inconstant signal amplitude, dynamic dc offset and phase ambiguity in large-scale displacement tracking. Experiments have been carried out in the outdoor environment to monitor the relative water level position in a rain barrel when the water was pumped in or drained out. It is shown that the proposed technique can accurately gauge the relative water level variation of 3.5 wavelengths with $\mathrm{mm}$ accuracy.
\end{abstract}

Index Terms - DC calibration, displacement measurement, large displacement, radar, water level.

\section{INTRODUCTION}

$\mathbf{M}$ ICROWAVE radar has become an attractive approach for noncontact displacement and distance measurement [1]. The conventional Doppler radar technique has been used for small-scale displacement detection, e.g., vital signs of respiration and heartbeat (amplitude is less than a few $\mathrm{cm}$ ) [2]. The small displacement is negligible compared to the distance between the subject and the radar. Therefore, the amplitudes of the measured signals and the dc offset at RF output are almost constant, and typically the phase modulation does not exceed $180^{\circ}$ (half carrier wavelength). Arctangent demodulation with pre-

Manuscript received July 14, 2014; accepted August 17, 2014. Date of publication September 11, 2014; date of current version December 01, 2014. This work was supported by the National Science Foundation (NSF) under grant CMMI-1131506, NSFC under grant 61131002 and the National Instruments.

C. Gu was with the Department of Electrical and Computer Engineering, Texas Tech University, Lubbock, TX 79409 USA and is now with Marvell Semiconductor Inc., Santa Clara, CA 95054 USA (e-mail: cgu@marvell.com).

$\mathrm{W} . \mathrm{Xu}$ is with the Department of Computer Science and Engineering, State University of New York (SUNY), Buffalo, NY 14260 USA (e-mail: wenyaoxu@buffalo.edu).

G. Wang and C. Li are with the Department of Electrical and Computer Engineering, Texas Tech University, Lubbock, TX 79409 USA (e-mail: guochao. wang@ttu.edu; changzhi.li@ttu.edu).

T. Inoue is with National Instruments, Austin, TX 78759 USA (e-mail: takao. inoue@ni.com).

J. A. Rice is with the Department of Civil and Costal Engineering, University of Florida, Gainesville, FL 32611 USA (e-mail: jrice@ce.ufl.edu).

L. Ran is with the Department of Information and Electronic Engineering, Zhejiang University, Hangzhou 310027 China (e-mail: ranlx@zju.edu.cn).

Color versions of one or more of the figures in this letter are available online at http://ieeexplore.ieee.org.

Digital Object Identifier 10.1109/LMWC.2014.2352852

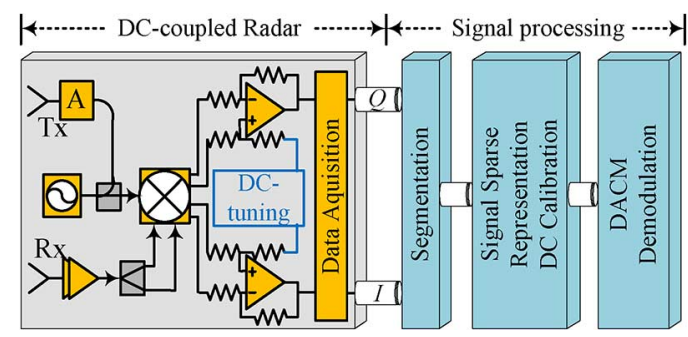

Fig. 1. Block diagram of the radar for large-scale displacement tracking.

fixed dc calibration is sufficient to recover the phase information of the small-scale displacement [2]. However, if the displacement is so large that it is comparable to the distance between the subject and the radar, e.g. in the case of radar water level gauging [3], it would significantly affect the power received at the radar input and the baseband $I / Q$ signals would be subject to inconstant amplitude and dynamically varying dc offset. Moreover, the large displacement inevitably leads to phase ambiguity in the conventional arctangent demodulation.

In this letter, a novel Doppler radar technique is presented for accurate tracking of large-scale displacement of several carrier wavelengths. Since AC-coupled radar architecture may lead to signal distortions [4], dc coupling is used to ensure precise measurement of slow motions even with stationary moment [4]. To tackle the issues of inconstant amplitude, dynamic dc offset and phase ambiguity, a novel signal processing approach is proposed to extend the demodulation linearity to large-scale displacement tracking. The approach is based on signal segmentation to generate stable signal cells, and two algorithms to automatically calibrate the dynamic dc offset and recover the phase information without phase ambiguity. The proposed radar technique for large-scale displacement tracking has been validated in an outdoor environment to monitor the relative water level position in a rain barrel when the water was pumped in or drained out. Compared with UWB and FMCW radars for water level gauging, the proposed Doppler radar avoids the bandwidth requirement to achieve desirable range resolution and can operate at lower frequencies with less cost. Fig. 1 shows the block diagram of the radar for large-scale displacement tracking.

\section{THEORY}

The radar baseband $I / Q$ outputs are digitized by a data acquisition card (USB6009) and they are:

$$
\begin{aligned}
& B_{I}[n]=A(n) \cdot \cos \left[\frac{4 \pi x(n)}{\lambda}+\Delta \varphi\right]+D C_{I}(n), n=1,2,3 \ldots \\
& B_{Q}[n]=A(n) \cdot \sin \left[\frac{4 \pi x(n)}{\lambda}+\Delta \varphi\right]+D C_{Q}(n), n=1,2,3 \ldots
\end{aligned}
$$




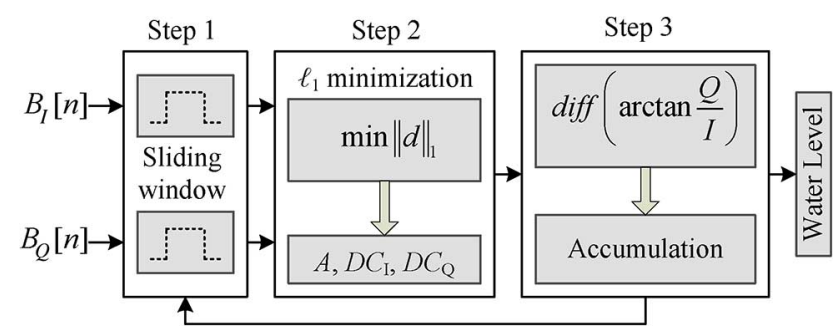

Fig. 2. Flow chart of the signal processing for water level gauging. Steps: 1) signal segmentation using a sliding window, 2) dc calibration based on SSR, and 3) phase demodulation based on DACM.

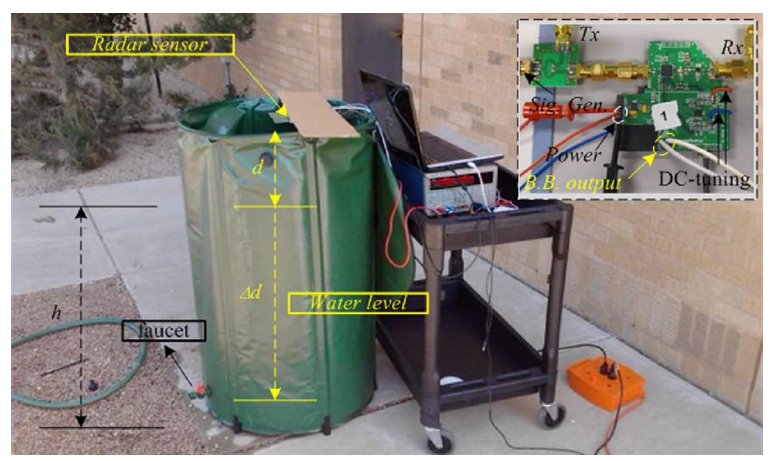

Fig. 3. Experimental setup of radar sensor measuring the water level in a rain barrel in an outdoor environment. Inset shows the designed DC-coupled radar.

where $x(n)$ is the varying water level, $\lambda$ is the wavelength of the carrier signal, $\Delta \varphi$ is the residual phase, $A(n)$ is the amplitude of the measured signal, and $D C_{I}(n) / D C_{Q}(n)$ are the dc offset of the $I / Q$ channels. Since the water level variation is so large that it is not negligible compared to the distance between the water level and the radar, the radar-measured signals would have distance-dependent amplitude modulation and time-varying dc offset, which makes it challenging to calibrate the radar signals. In this case, the conventional arctangent demodulation with prefixed dc offset calibration would not be suitable. To precisely measure large displacement of water level, a novel signal processing approach has been proposed, as shown in Fig. 2. There are three steps in the signal processing flow: 1) signal segmentation, 2) dc calibration, and 3) phase demodulation.

Step 1: The measured signals are divided into a sequence of fixed-length window cells whose length is much smaller than the signal period. Therefore, the amplitude change within one window cell is minimal. It is assumed that the amplitude within the window cell is stable and dc offset values will not change as well. The dc offset calibration and phase demodulation will be performed in Step 2 and Step 3, respectively. Then it will move to the next window cell. This process continues until the end of the segments. The overall water level information is reconstructed by combining the displacement recovered in phase demodulation from each segment.

Step 2: From (1) and (2), it is seen that each $I / Q$ measurement, $\left\langle B_{I}[i], B_{Q}[i]\right\rangle$, will sit on a circle centered at $\left(D C_{I}, D C_{Q}\right)$ with a radius of $A$. Therefore, the $\mathrm{dc}$ offset calibration problem can be formulated as follows: given a set of $I / Q$ quadrature signals with $n$ samples:

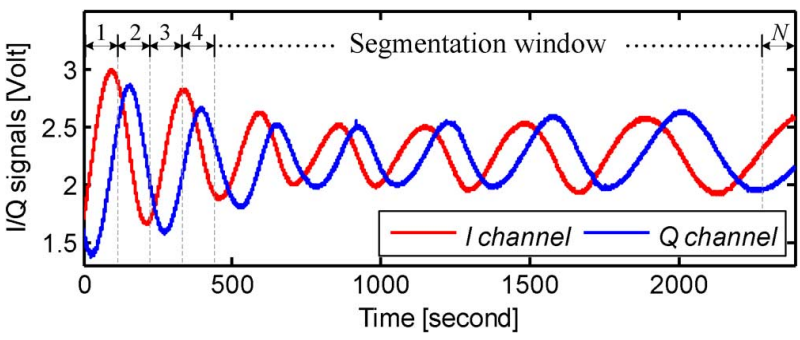

Fig. 4. Radar measured time-domain $I / Q$ signals with varying amplitude. Signal segmentation is applied to make the signal amplitude relatively constant within each segmentation window.

$\left\langle B_{I}(1: n), B_{Q}(1: n)\right\rangle=\left\{\left(I_{1}, Q_{1}\right),\left(I_{2}, Q_{2}\right) \ldots\left(I_{n}, Q_{n}\right)\right\}$, there is a tuple $\left(D C_{I}, D C_{Q}, A\right)$ such as

$$
\begin{aligned}
& \min \|d\|_{2} \\
& d=\left[d_{1}, d_{2} \ldots d_{i} \ldots d_{n}\right], \\
& d_{i}=\left(I_{i}-D C_{I}\right)^{2}+\left(Q_{i}-D C_{Q}\right)^{2}-A^{2}
\end{aligned}
$$

where $d_{i}$ is the fitting residual between the measurement $\left(I_{i}, Q_{i}\right)$ and the circle $\left(D C_{I}, D C_{Q}, A\right)$. Therefore, according to (3) and (4), the dc offset calibration is formulated into a least square (LS) optimization problem, i.e. norm-2 $\left(\ell_{2}\right)$ minimization [5]. The LS method will try to minimize the root mean square error (RMS) out of all measurements regardless of considering measurement errors. These outliers will affect the accuracy of the dc offset estimation. In this part, a new heuristic is proposed to reduce the number of measurements far away from the fitting circle. It pushes every residual item $d_{i}$ to zero and minimizes the number of non-zero items in $d$. Note that the value of non-zero items can be large, and it is different from RMS error minimization. This heuristic is called sparsity pursuit and can be formulated with the norm-0 $\left(\ell_{0}\right)$ of $d$ :

$$
\min \|d\|_{0}
$$

(5) is the norm- $0\left(\ell_{0}\right)$ minimization problem and belongs to intractable NP-hard problems. However, it is proved that the solution in (5) is the same as the solution in norm- $1\left(\ell_{1}\right)$ with very high probability, which is a linear programming problem [5]. The final form of dc offset calibration can be presented as

$$
\min \|d\|_{1}
$$

It is seen that (6) is a well-posed problem and can be solved efficiently. Specifically, the solution of (6) is to obtain the sparsest representation of fallacious measurements.

Step 3: In arctangent demodulation, the phase discontinuity happens when the demodulation exceeds the native range of $(-\pi / 2, \pi / 2)$. Although it could be eliminated theoretically by shifting the discontinuous points by an integer multiple of $\pi$, it is actually difficult for a hardware to automatically make an judicious choice on where to compensate the phase, especially in real-time applications where segmentation window may change the length to accommodate the pumping/draining speed. An extended differentiate and cross-multiply (DACM) algorithm is employed for automatic phase unwrapping for phase reconstruction without ambiguities [6]

$$
\phi(t)=\arctan \left[\frac{Q(t)}{I(t)}\right]
$$



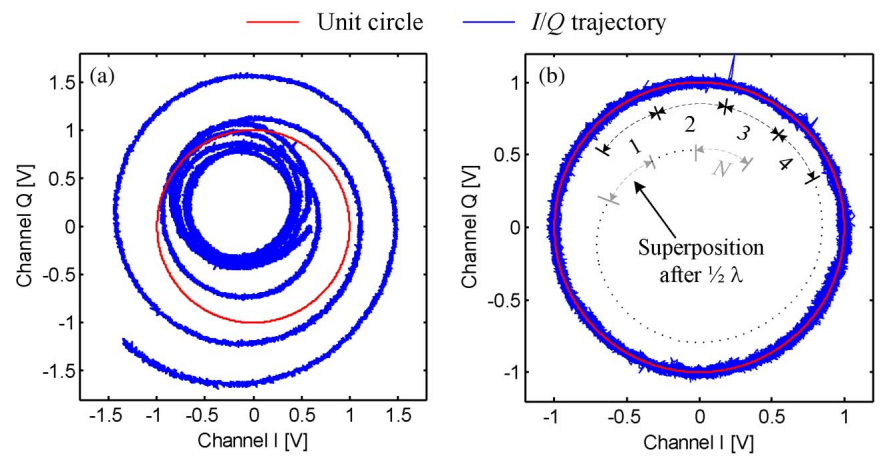

Fig. 5. Trajectories consisting of $I / Q$ signals: (a) original signals with prefixed dc compensation, and (b) after signal segmentation and dc calibration based on SSR. Trajectory superimposition happens after half a wavelength.

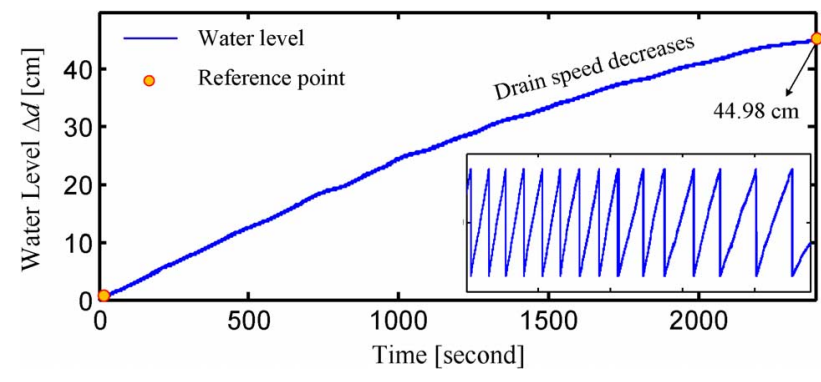

Fig. 6. Radar measured water level. The inset shows the demodulated water level signal using the conventional arctangent demodulation.

TABLE I

Summary of 10 Measurements (No.1-5: Drain Out, No.6-10: PumP IN)

\begin{tabular}{|c|c|c|c|c|c|c|c|}
\hline No. & Meas. [mm] & Ref. [mm] & Err. [mm] & No. & Meas. [mm] & Ref. [mm] & Err. [mm] \\
\hline 1 & 449.8 & 450.0 & 0.2 & 6 & 224.5 & 226.0 & 1.5 \\
\hline 2 & 373.6 & 372.5 & 1.1 & 7 & 459.9 & 460.5 & 0.6 \\
\hline 3 & 420.3 & 421.0 & 0.7 & 8 & 403.5 & 402.5 & 1.0 \\
\hline 4 & 256.3 & 255.0 & 1.3 & 9 & 353.8 & 355.0 & 1.2 \\
\hline 5 & 345.9 & 346.5 & 0.6 & 10 & 320.4 & 320.0 & 0.4 \\
\hline
\end{tabular}

where $I(t) / Q(t)$ are $I / Q$ signals after calibration. With a further accumulation, the phase information $\phi[n]$ is reconstructed

$\phi[n]=\sum_{k=2}^{n} \frac{I[k]\{Q[k]-Q[k-1]\}-Q[k]\{I[k]-I[k-1]\}}{I[k]^{2}+Q[k]^{2}}$.

Instead of involving any phase unwrapping procedures, the DACM based phase demodulation technique allows the water level information to be directly retrieved from the calibrated $I / Q$ signals without any ambiguity.

\section{EXPERIMENT}

As shown in Fig. 1, the DC-coupled radar sensor has been integrated with the signal processing approach and it can work in a wide range of $300 \mathrm{MHz}$ to $4 \mathrm{GHz}$, which helps suppress the water ripples by properly choosing the carrier frequency [3]. The experimental setup is shown in Fig. 3. The inset shows the designed DC-coupled radar sensor. A rain barrel was filled with water with a depth of $h=75 \mathrm{~cm}$. The designed radar sensor was placed over the rain barrel, facing the water surface at a distance of $d=25 \mathrm{~cm}$. The radar sensor was configured to work at $2.4 \mathrm{GHz}$ and measured the water level when the water drained out slowly (water level dropped a relative distance of $\Delta d$ ). The initial position of the water level was marked on the rain barrel and the end position was also recorded so as to provide a reference for the water level drop.

The radar measured water level signals are shown in Fig. 4. It is seen that the measured signals are periodic but have varying amplitude. It is because the water level drops as time elapses. The received signal is amplitude-modulated due to this detection distance change. It shows stronger signals at the end of the time, due to the stronger reflections from the bottom of the barrel as the water level drops. However, the stronger time domain signals do not affect the measurement accuracy because the water level information is modulated in the phase.

The trajectory of the original $I / Q$ signals with pre-fixed dc compensation is illustrated in Fig. 5(a), which shows an irregular helical shape. In contrary, Fig. 5(b) shows the $I / Q$ trajectory using the proposed signal processing approach. It is seen that it fits the unit circle well. Each segment forms a short arch on the unit circle, and all the arches are connected to form a full unit circle. It is noted that the arch superposition happens after the water level drops over a half wavelength of the radar carrier, because the phase modulation exceeds $360^{\circ}$.

The water level information demodulated by the proposed signal processing approach is shown in Fig. 6. It is seen that the phase information has been reconstructed well without any phase ambiguity, while the arctangent demodulation leads to phase discontinuity, as shown in the inset of Fig. 6. It is also seen that the slope of the water level decreases as time elapses, because the water pressure at the faucet decreases as the water level drops, which leads to slower drawdown speed. The radar measured water level variance is $44.98 \mathrm{~cm}$ and the reference level drop is $45 \mathrm{~cm}$. Measurements were also performed for different water levels when water was pumped in or drained out. Table I shows the data summary, which indicates $\mathrm{mm}$ accuracy. In order to detect absolute distance from radar to water surface, calibration needs to be done before the measurement.

\section{CONCLUSION}

A Doppler radar technique has been proposed for noncontact detection of large-scale displacement of a few carrier wavelengths. A novel signal processing approach has been designed to deal with the varying signal amplitude and dynamic dc offset to recover large phase variation. Experiments were carried out to validate that the proposed radar technique is able to gauge the water level with $\mathrm{mm}$ accuracy.

\section{REFERENCES}

[1] S. Kim and C. Nguyen, "A displacement measurement technique using millimeter-wave interferometry," IEEE Trans. Microw. Theory Tech., vol. 51, no. 6 , pp. 1724-1728, 2003.

[2] B. K. Park, O. Boric-Lubecke, and V. M. Lubecke, "Arctangent demodulation with DC offset compensation in quadrature Doppler radar receiver systems," IEEE Trans. Microw. Theory Tech., vol. 55, no. 5, pp. 1073-1079, 2007.

[3] G. Wang, C. Gu, J. Rice, T. Inoue, and C. Li, "Highly accurate noncontact water level monitoring using continuous-wave Doppler radar," in IEEE Topical Conf. Wireless Sensors Sensor Netw. (WiSNet'13), Jan. 20-23, 2013, pp. 19-21.

[4] C. Gu, R. Li, R. Fung, C. Torres, S. Jiang, and C. Li, "Accurate respiration measurement using DC-coupled continuous-wave radar sensor for motion-adaptive cancer radiotherapy," IEEE Trans. Biomed. Eng., vol. 59, no. 11, pp. 3117-3123, Nov. 2012.

[5] W. Xu, C. Gu, C. Li, and M. Sarrafzadeh, "Robust Doppler Radar Demodulation via Compressed Sensing," Electron. Lett., vol. 48, no. 22, pp. $1428-1430$, Oct. 2012.

[6] H. Bukac, "Instantaneous frequency: Another tool of source of noise identification," in Proc. Int. Compressor Engr. Conf., Jul. 2004, [CD ROM]. 